Research Article

Elena Pavlova* and Viktor Voropaev

\title{
Prevention of NEO hazard: the Russian approach
}

https://doi.org/10.1515/astro-2021-0007

Received Sep 08, 2021; accepted Oct 18, 2021

\begin{abstract}
Russian Academy of Sciences (RAS) is actively working to solve the problem of preventing Near-Earth Object (NEO) hazard. The article provides the goals and objectives of preventing NEO hazard, as well as ways to achieve them. The described ways of achieving the goal are applied in RAS best. The current state and prospects of the Russian contribution to the prevention of NEO hazard are considered. Statistical data on the dynamics of observation of minor planets by Russian telescopes is presented. The structure of the NEO part of hazardous situations classifier developed by Keldysh Institute of Applied Mathematics (KIAM RAS) and structure of perspective National System Security of space activities in outer space are presented, including the system's response to event classified as dangerous. The article is accompanied by illustrations of potentially hazardous objects in the sky, made by telescopes of the International Network of Optical Telescopes (ISON), coordinated by the KIAM RAS.
\end{abstract}

Keywords: asteroids, space security and sustainability, KIAM, ISON, telescopes, national security system, observatories, telescopes, space activities

\section{Introduction}

Reliable and timely warning of NEO hazard is one of the modern challenges of humankind. This is a global element of ensuring the sustainability of space activities. At present, the Russian approach in case of NEO hazard has moved from a theoretical plane to a practical one.

Scientists from Russian institutes conduct research on different aspects related to detection and prevention of NEO hazard. Over the past decade, scientists from KIAM RAS have catalogued dozens of new main belt asteroids and discovered several potentially dangerous asteroids and comets. In 2020-2021 during the International Asteroid Warning Network (IAWN) campaign KIAM's specialists obtained a good volume of positional and photometric data on the asteroid 99942 Apophis.

Positive results of this activity is indicate the right direction of Russian approach for prevention of NEO hazard and indicate the existence of their own national approaches to solving these challenges. However, it is important to organize the national system of security that is able to respond to NEO hazard. The system should follow the goals of NEO prevention and solve the complex tasks associated with each of the directions.

Corresponding Author: Elena Pavlova: KIAM RAS, Miusskaya sq., 4, 125047, Moscow, Russia; Email: elenae312@gmail.com Viktor Voropaev: KIAM RAS, Miusskaya sq., 4, 125047, Moscow, Russia

ə Open Access. ๑ 2021 E. Pavlova and V. Voropaev, published by De Gruyter. Attribution 4.0 License

\section{The Russian approach to challenges for prevention of NEO hazard: goals and objectives}

Goals for prevention of NEO hazard determine the events that have occurred. For example, the event that occurred on February 15, 2013, over Chelyabinsk showed that when even a small object (about 15 meters in size) enters the atmosphere over densely populated areas of the Earth, serious consequences for the population can ensue in the form of injury from the impact of the shock wave. Such wave causes damage to buildings, as well as from the outbreak of panic due to the occurrence of an unanticipated event that outwardly resembles an accident caused by human beings or the use of nuclear weapons. So, the goals of NEO prevention should be aimed at ensuring the safety of the population, humankind, and infrastructure.

The goals and objectives of NEO prevention are following:

- timely detection of an object approaching the Earth;

- calculation and prediction of the trajectory of its motion;

- determination of the physical properties of the asteroid;

- assessment of accordance with the hazard criteria.

The solution of NEO hazard warning problem has a systematic nature. If the near-Earth object threatens to fall 
to the Earth, the most important task is to determine the time and coordinates of the place of possible fall. However, in order to solve the listed tasks and reach the goals, the elements of the NEO prevention system should be wellcoordinated. In our opinion, the constituent elements of the approach to the organization of a system when the system can respond quickly to assigned tasks are following:

- technical means (optical telescopes, radars, etc.) that can enable the planning of observations, detection and tracking of potentially dangerous object - minor planets and comets;

- advanced mathematical methods for solving astroballistic problems associated with determining the trajectory and calculating possible fall zones;

- maintenance of a database on the orbital and physical characteristics of objects of natural origin in nearearth space, including potentially dangerous asteroids and comets;

- the classification of NEO hazard in order to determine the criteria and degree of risks and probable consequences.

That will make it possible to expect the emergence and effective use of methods to counter them. The next section of the article describes tools and methods for prevention NEO hazard used in KIAM RAS.

\section{Tools and methods for prevention NEO hazard by KIAM RAS}

Our engineers and scientists use advanced technical, mathematical methods and scientific achievements to prevent NEO hazard.

The main technical means for prevention is optical telescopes. KIAM RAS owns and manages the International Network of Optical Telescopes (ISON) (see Figure 1).

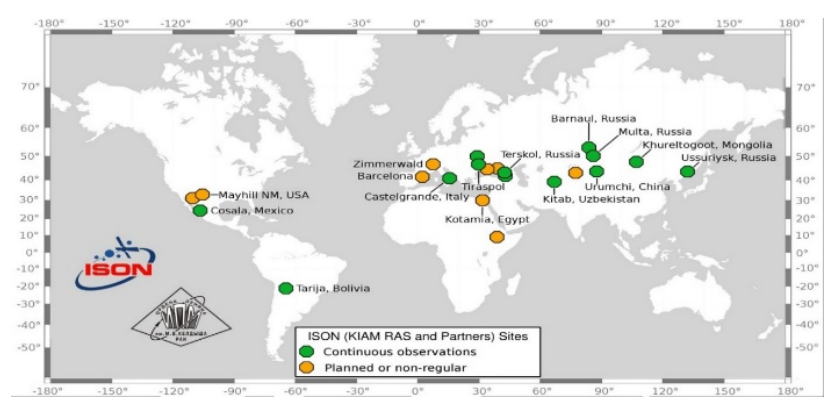

Figure 1. The geographic map of main KIAM/ISON facilities
Telescopes that observe asteroids within ISON initiatives are located worldwide, including Castelgrande (Italy) - see Figure 2, Multa (Altay region) - see Figure 3, Krasnodar (Russia), Tarija (Bolivia), Kitab (Uzbekistan), Simeiz (Crimea), Nauchny (Crimea), Khureltogoot (Mongolia) and Abastumani (Georgia).

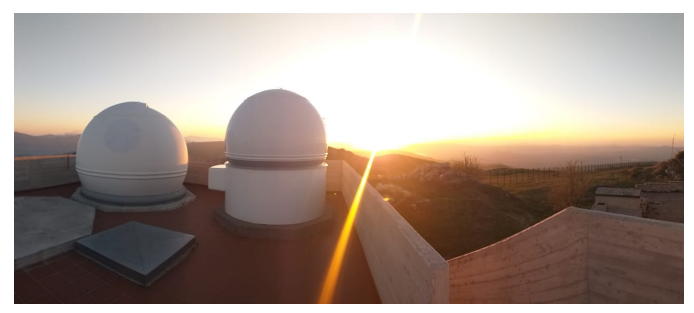

Figure 2. The sunset over KIAM / GAUSS Srl joint observatory in Castelgrande, Italy

The newest telescopes in this network are well automated and remotely controlled by observers. At the same time, the initial image processing is usually carried out on-site, which avoids a lot of traffic.

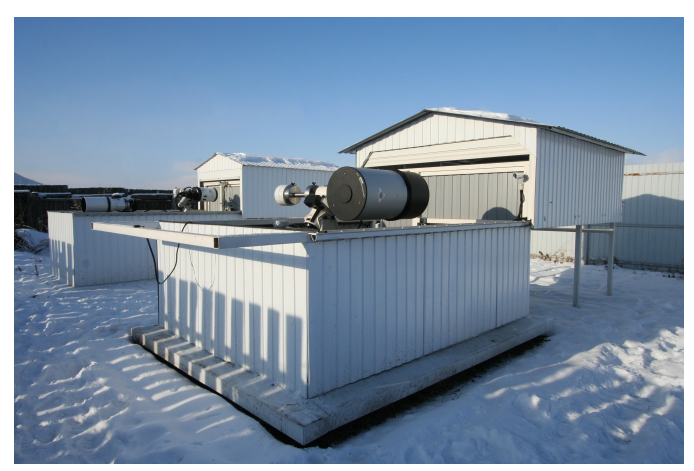

Figure 3. KIAM's $40 \mathrm{~cm}$ telescope in Multa, Altai region

The information received from the telescopes is collected in dedicated database stored in KIAM server.

The most important condition for effective prevention of NEO hazards is development and application of advanced mathematical methods. The results of reach a goal of leading KIAM's scientists are presented in numerous scientific publications.

For example, Prof. Vyacheslav V. Ivashkin with his pupils published a number of articles analyzed of the possibility of creating a stable satellite about the asteroid 99942 Apophis as a homogeneous triaxial ellipsoid. The task of creating a stable orbit of its satellite as a homogeneous triaxial ellipsoid is investigated. The simulation of the motion of the spacecraft around the asteroid was performed taking 
into account the main perturbations: from the attraction of celestial bodies, asteroid's non-sphericity and solar radiation pressure, and taking into account the asteroid's own rotation. It is shown that it is possible to create a stable orbit of an asteroid satellite (see Figure 4). This orbit can be used for a detailed study of the characteristics of the asteroid and the refinement of its orbit using ground radio measurements of the motion of a satellite equipped with a radio beacon (Ivashkin and Guo 2019).

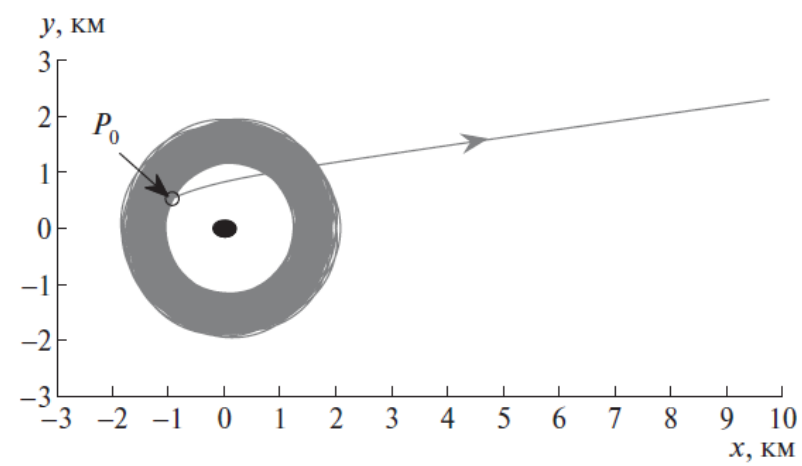

Figure 4. The orbital motion of the mini-satellite in the plane orthogonal to the asteroid-Sun direction (Ivashkin and Guo 2019).

One of the newest methods proposed to solve a global problem is using of the classifier. This method was proposed by one of the authors of this article (Pavlova et al. 2021). Hazardous events caused by NEO hazard can be divided into projected and unpredictable (depending on the reliability of the ballistic forecast of the movement, the lead interval) and into observed and unobserved (depending on the control by optical or radar observation means). Objects that completely burn up in the Earth's troposphere are accompanied by the threat of a shock wave, and those that partially burn up in the atmosphere can be divided into objects with a calculated drop zone or without it, depending on the motion trajectory. The basic scheme of classification of this type of threat is shown in Figure 5.

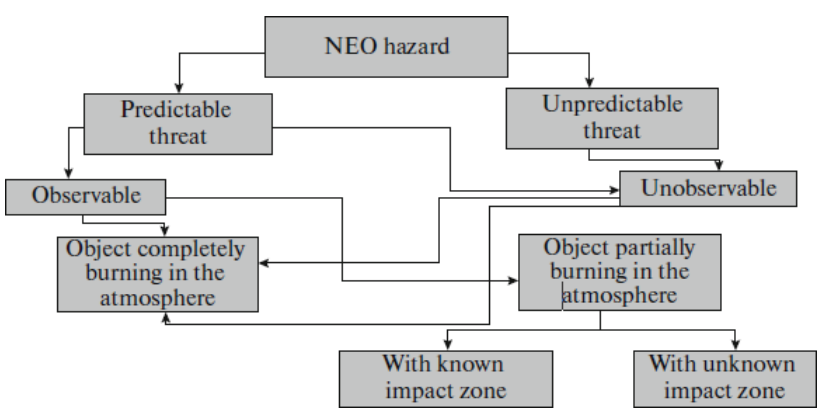

Figure 5. Basic classification of NEO hazard (Pavlova et al. 2021).
The system's response to event classified as a NEO hazard may be as follows: for example, the threat is unpredictable - newly identified, but the asteroid is far away, we noticed it early - we are observing it. The classifier noted us that the object is large, and when entering the Earth's atmosphere, it will not be able to completely burn up in its dense layers. But since the object was seen at a great distance from the Earth, the fall zone is calculated. The system issues a message about the occurrence of a situation classified as an NEO hazard (see Figure 6).

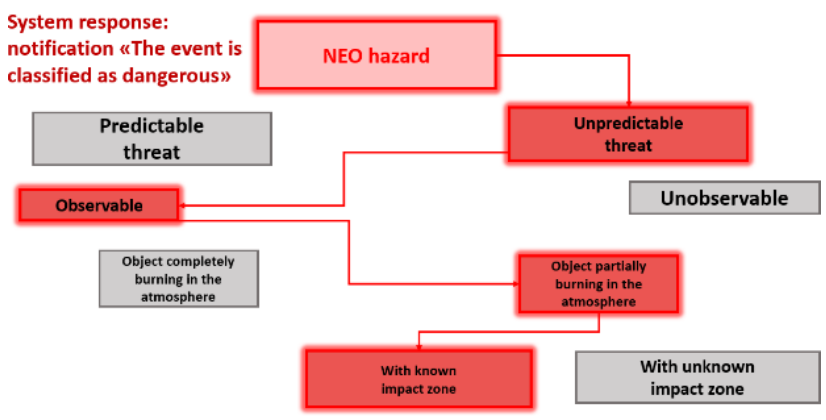

Figure 6. Example of the system's response to event classified as dangerous.

However, it's important to properly build the architecture of a promising National Security System of space activities in outer space (NSS system) for effective parrying any dangerous event including NEO hazard. The author of the article proposes a diagram of the interaction of the main centers of security system in the event of a dangerous situation (see Figure 7).

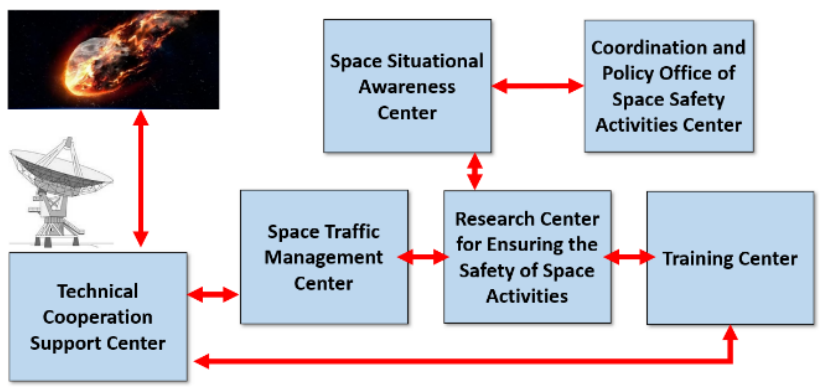

Figure 7. The diagram of the interaction of the main centers of the NSS system in the event of a dangerous situation.

The system architecture is universal structure. The system should address the challenges of ensuring safety in near-Earth space and dangerous situations in outer space, including prevention NEO hazard. 
Main actors of such a system are dedicated centers. These are the following:

\section{Technical cooperation support center}

This center must include any technical instruments for observing different objects in outer space. In the event of a dangerous situation, the control facilities of this center should bring the primary information about the detected object to the control services on space traffic management center.

\section{Space traffic management center}

This center should be able to control spacecrafts. It can verify the detection of a new asteroid by the measurement and astroballistic data of the research center for ensuring the safety of space activities. The result of the joint processing of all available data should be transmitted from the space traffic management center to the space situational awareness center.

\section{Space situational awareness center}

It should be used to any kind of space situation analysis and to assess the risks of a catastrophic situation. If necessary, it brings information to the coordination and policy office of space safety activities center.

Research center for ensuring the safety of space activities This is a scientific community. The results of functioning the NSS system, including organizational and technical documentation obtained during the detection and prevention of hazardous situation must be sent to the training center for inclusion in the curricula aimed at obtaining solid practical skills to counter future threats.

\section{Training center}

This center should give knowledges to rising specialists.

\section{Coordination and policy office of space safety activities center}

This center should make decisions about the need to counter the emerging threat and inform the Russian Government and foreign countries about the emerging threat.

In order to achieve the goals and objectives of the NSS system should include structures formed under the authorized federal bodies, organizations and enterprises of the space industry. In the structures of organizations, min- istries and departments proposed to form specialized centers, their interaction should become the basis for formation of the NSS system. The system should be designed in such a way as to ensure maximum interaction between government departments and to respond promptly to dangerous events.

It's great important to consolidate the efforts of the Russian Academy of Sciences, Roscosmos State Corporation's enterprises, the Ministry of Defense of the Russian Federation, the universities, the Government of the Russian Federation, and other state structures. Unfortunately, we don't have that in the Russian Federation right now.

\section{Practical results of prevention NEO hazard}

The next important aim of this article is to note some the practical results of prevention NEO hazard that have been achieved so far and these results were achieved only through the efforts of the Russian Academy of Sciences.

In the Russian Federation, complex studies of nearEarth asteroids are carried out, including the study of their physical properties and astroballistic issues. For example, Institute of Applied Astronomy RAS uses the method of receiving and analyzing radar signals using radio telescopes of the own Quasar VLBI network to study near-Earth asteroids (see Figure 8).
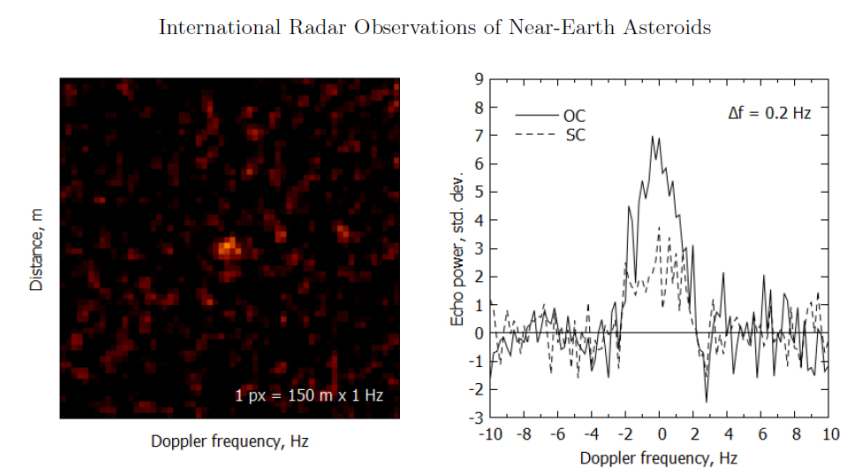

Figure 8. International Radar Observations of Near-Earth Asteroids on Radio Telescopes of the Quasar VLBI Network (Bondarenko et al. 2020).

To date, six Russian research institutes are members of the IAWN established as the initiative of the UNOOSA: Crimean Astrophysical Observatory, Kourovka Astronomical Observatory, Special Astrophysical Observatory, 
Keldysh Institute of Applied Mathematics, Institute of Astronomy, and Institute of Solar-Terrestrial Physics.

During the latest IAWN campaign dedicated to observing the potentially dangerous asteroid 99942 Apophis, that flew near Earth in March 2021, data obtained by the ISON/KIAM telescope were used. This campaign made it possible to identify weaknesses in the technology of timely detection of dangerous asteroids and improve existing methods and tools, including software.

The diagram of the statistics of asteroid observations by Russian observatories and its neighbors is seen in Figure 9.

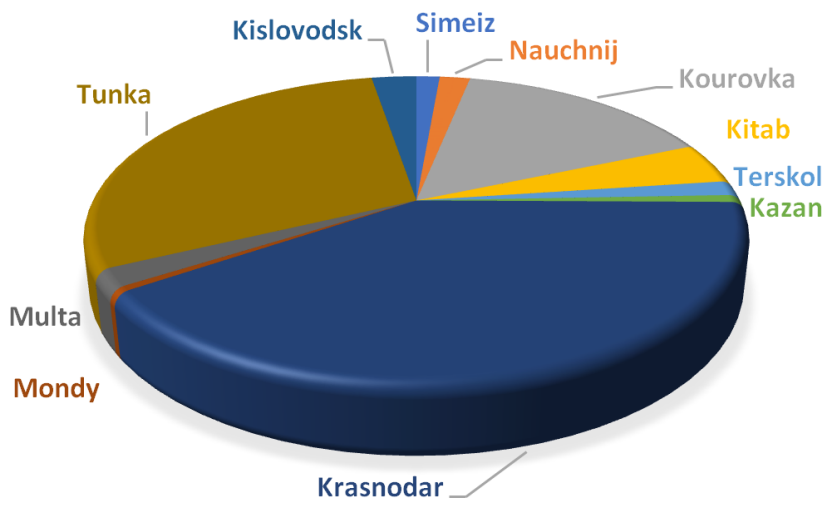

Figure 9. Statistics of Minor Planet measurements by Russian telescopes in 2021.

As we can see the statistics from Figure 9, Kuban State University in Krasnodar is the current leader of KIAM's NEO research collaboration. This observatory conducts specialized observations of asteroids in joint programs with KIAM every clear night and it's the big contribution for prevention of NEO hazard.

The main instrument of the observatory is the $50 \mathrm{~cm}$ telescope manufactured by Astrosib, Russia. The telescope is located inside the upper dome in Figure 10.

Under favorable weather conditions, typical $40-\mathrm{cm}$ telescopes can observe near-Earth asteroids as a quite dim objects with a brightness up to $19^{m}$.

All data from Russian telescopes is promptly transmitted to the Minor Planet Center at Harvard University, USA. The real NEO image obtained by the KIAM/ISON collaborator's telescope is shown in Figure 11.

The methods and approaches described above to solving the tasks of monitoring, warning and - as we hope - to parrying the NEO threat in the near future allow us to count on Russia's worthy place in these worldwide processes.

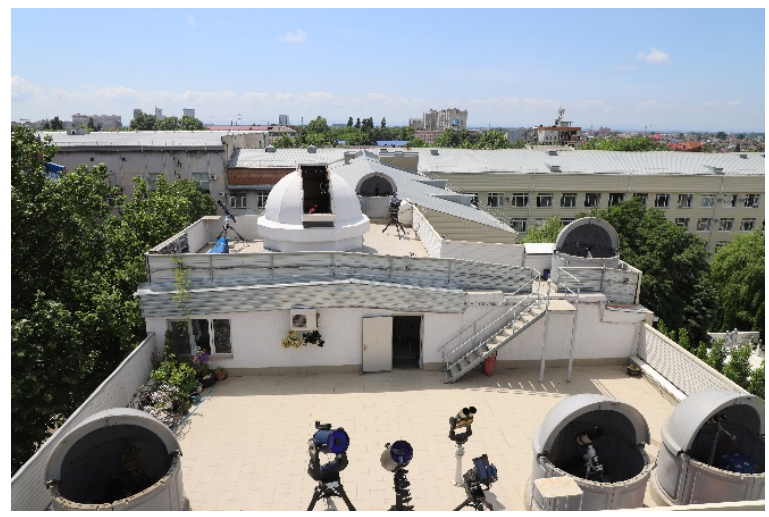

Figure 10. The Kuban State University Observatory.

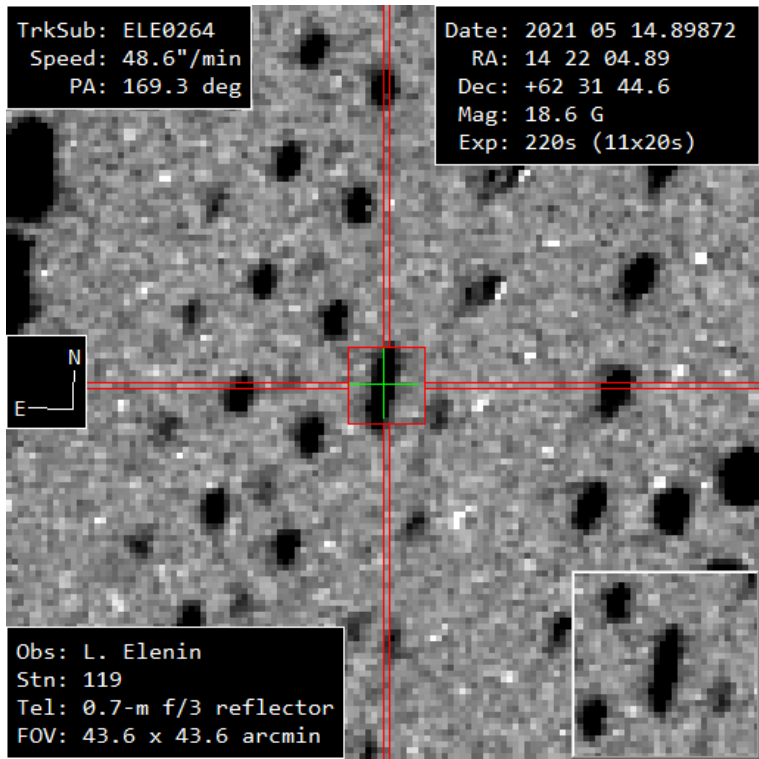

Figure 11. Near-Earth Asteroid $2015 \mathrm{KJ} 19$ tracked by 70-cm telescope AC-32, AbAO.

\section{Conclusion}

Challenges of prevention of NEO hazard have a complex systemic nature. Most important means and methods to solve this task are technical means, computer systems, advanced mathematical methods, and software solutions. These elements must be interconnected in a single structure to achieve the goal of warning NEO hazard.

Prevention of NEO hazard is one of the modern challenges of humankind. The Russian Federation itself has a huge potential for preventing NEO hazard. To date this activity has moved from a theoretical plane to a practical one by the efforts of Russian Academy of Sciences only.

Acknowledgment: Part of NEO observations mentioned above were performed at telescope Zeiss-1000 of Simeis 
Observatory as shared research facility "Terskol observatory" of Institute of Astronomy of the Russian Academy of Sciences.

Funding information: The authors state no funding involved.

Author contributions: All authors have accepted responsibility for the entire content of this manuscript and approved its submission.

Conflict of interest: The authors state no conflict of interest.

\section{References}

Bondarenko Y, Marshalov D, Vavilov D, Medvedev Y. 2020. International radar observations of near-Earth asteroids on radio telescopes of Quasar VLBI Network. Ground-Based Astronomy in Russia. 21st Century, Proceedings of the All-Russian Conference 455-457.

Ivashkin V, Guo P. 2019. Analysis of the possibility of creating a stable satellite about the asteroid Apophis as a homogeneous triaxial ellipsoid. Rep of RAS. 489:27-33.

Pavlova E, Zakhvatkin M, Streltsov A, Voropaev V, Elenin L. 2021. Development of the Common Classification of Hazard Events in Near-Earth Space. Cosmic res. 59(2):104-111. 